\title{
Role of CBCT in Detection of Osteomyelitis of Mandible: A Case Report
}

\author{
Ashwini Pai, BDS ${ }^{\text {a }}$; Nikhil Diwan, MDS ${ }^{\text {B }}$; R.Krishna Kumar MDS ${ }^{\mathrm{C}}$; Amita \\ Aditya MDS ${ }^{\mathrm{d}}$; Rashmi Sapkal ,MDS ${ }^{\mathrm{E}}$; Kunal Advani , MDS ${ }^{\mathrm{f}}$ \\ ${ }^{A}$ post Graduate Student, Department Of Oral Medicine And Radiology, MARDC, \\ ${ }^{B}$ reader, Department Of Oral Medicine And Radiology, M.A.Rangoonwala Dental College \& \\ Research Center, Pune (MARDC), \\ ${ }^{C}$ Professor \& HOD, Department Of Oral Medicine And Radiology, MARDC, \\ ${ }^{D}$ reader, Department Of Oral Medicine And Radiology, Sinhgad Dental College \& Hospital, Pune \\ ${ }^{E}$ reader, Department Of Oral Medicine And Radiology, MARDC; ${ }^{F}$ Oral Medicine And Radiologist ;
}

\begin{abstract}
Osteomyelitis is a true infection of the bone induced by pyogenic microorganisms. Other than chronic cases, conventional imaging modalities do not offer much radiographic details. Computed tomography is considered the best modality for imaging the osteomyelitic cases. However, CBCT provides a lower dose, lower cost alternative to conventional CT and has gained increased acceptance as a 3D imaging modality.CBCT has potential to sufficiently depict osteomyelitic lesions like osteolytic and osteosclerotic areas, ill-defined cortical borders with periosteal reaction and sequestra.

We present a case report of osteomyelitis in which periosteal reaction and sequestra is well appreciated in all three sagittal, coronal and axial sections of CBCT and reemphasizes the role of CBCT as a useful tool in the field of diagnostic maxilla-facial Radiology.
\end{abstract}

\section{Introduction}

The word "osteomyelitis" originates from the ancient Greek words osteon (bone) and muelinos (marrow) and means infection of medullary portion of the bone ${ }^{[1]}$ It is more commonly seen in the mandible than the maxilla because of the dense, poorly-vascularized cortical plates and the only blood supply from the inferior alveolar neurovascular bundle. ${ }^{[2]}$ The term "osteomyelitis" is used to describe a true infection of the bone induced by pyogenic microorganisms, although other etiological factors, such as traumatic injuries, radiation, may also produce inflammation of the medullary space. ${ }^{[3]}$

Jacobsso et al characterized the radiological findings as cortical destruction, osteolytic areas in sclerotic zones, dissolution, loss of trabecular pattern, moth eaten appearance, and shortening of tooth roots. ${ }^{[4]}$ Features directly linked to osteomyelitis are periosteal bone formation and sequestra. Bone changes with respect to inflammatory diseases require a significant loss of bone mineral content to be radiologically evident. Therefore, negative radiological findings do not exclude acute osteomyelitis. Plain film radiography is helpful only in chronic conditions where the moth eaten appearance of bone is evident after considerable osteolytic activity. ${ }^{[5]}$ The use of bone window $\mathrm{CT}$ enables high quality imaging of osteomyelitis and is able to define the precise location and extent depending on the appearance of the fascial spaces. ${ }^{[6]}$

Cone-beam computed tomography (CBCT) has gained increased acceptance as a 3D imaging modality, offering an alternative to CT especially in the maxillofacial area. ${ }^{[7]}$ High-contrast structures can be visualized almost equal to bone window CT combined with a reduced radiation exposure ${ }^{[8]} \mathrm{CBCT}$ sufficiently depicts osteomyelitic lesions like osteolytic and osteosclerotic areas, ill-defined cortical borders with periosteal reaction and sequestra. ${ }^{[5]}$

\section{Case Report}

A male patient aged 42 years reported to the department of Oral Medicine and Radiology with a painful and persistent bony hard swelling with an extra oral sinus tract involving the left lower border of mandible since 3 months. [Figure 1a] Extra oral drainage of pus was carried out a month ago at a general hospital. As the investigation of oral cavity for the foci of infection was ignored earlier, the extra oral sinus persisted.

The swelling was diffuse, bony hard and tender extending from mid cheek region to $2 \mathrm{~cm}$ below the lower border of mandible superio-inferiorly and $1 \mathrm{~cm}$ away from corner of mouth to angle of mandible anteroposteriorly with an extra-oral draining sinus at the lower border of mandible in the region of 37. Localized ipsilateral submandibular lymphadenopathy was noted. Intraoral examination revealed root pieces with $37 \& 38$. Based on these findings provisional diagnosis of chronic alveolar abscess was made. An intra oral periapical 
radiograph revealed root piece of 38 , a submerged and carious 37 with acutely dilacerated roots and an illdefined periapical radiolucency involving 36, 37 [Figure 1b]

Cone Beam CT imaging was carried out to evaluate the extent of the pathology and the submerged tooth's proximity to the associated structures. The reconstructed Panoramic image on the CBCT revealed an osteolytic lesion. Sagittal and coronal sections showed sequestra and perforation of buccal and lingual cortex. [Figure 2] Coronal and Axial sections revealed the periosteal reaction. [Figure $3 \&$ Figure 4] The inferior alveolar nerve canal was pushed lingually by the lesion. Complete haemogram was done and it showed an increase in WBC count. The history of a slow progressive swelling with clinical findings of a bony hard, diffuse swelling with extraoral draining sinus and typical radiographic findings of formation of sequestrum, perforation of buccal and lingual cortex and periosteal reaction led to a final diagnosis of Chronic Suppurative Osteomyelitis.

The patient was then referred to the Department of Oral and Maxillofacial Surgery for further treatment. Initial surgical treatment consisted of removal of offending teeth, sequestrectomy and decortication in conjunction with intravenous antimicrobial therapy. There was satisfactory healing and resolution of the extra oral sinus tract upon a one week recall.

\section{Discussion}

A popular imaging approach for dental and maxillofacial surgery practices has been Panoramic radiography. However, it creates only flattened out, two dimensional images with ghost images and a definite superimposition of all the surrounding structures. Additionally, bone density has to be reduced by $30-50 \%$ to be visible in plain radiography and this usually takes $2-3$ weeks in acute osteomyelitis. ${ }^{[9]}$

In our case, conventional IOPA and panoramic radiography did not reveal any osteomyelitic features. Sequestrum, perforation of cortex and periosteal reaction was only visible after taking a CBCT scan with a small FOV of the selected region. CBCT allows these anatomic entities to be viewed in three dimensions creating images that are both dimensionally faithful and anatomically accurate without distortion, magnification and superimposition. ${ }^{[10]}$

The importance of imaging in osteomyelitis is to localize the condition, to find out its extent, and to assess the response after treatment. Conventional CT is a useful method of preoperative assessment. ${ }^{[11]}$ However, CBCT provides a lower dose, lower cost alternative to conventional CT and promises to improve the practice of oral and maxillofacial radiology. ${ }^{[12,13]}$

Irregular margins are a common radiographic trait of malignancy. Interestingly, osteomyelitis has similar irregular margins. A malignant lesion is less likely to foster a new layer of periosteal bone, while in contrast chronic infection frequently exhibits such layering. Such radiographically similar lesions of widely different prognosis can be differentiated on the basis of periosteal reaction and cortical destruction. With multiplanar slices of a CBCT scan thin layers of periosteal reaction is better viewed compared to occlusal radiographs. ${ }^{[14]}$ In addition, small bony sequestra associated with osteomyelitis are better identified with crosssectional imaging as shown in our case.

\section{Conclusion}

In conclusion, two dimensional plain radiography techniques may be a hindrance in accurate diagnosis of maxillofacial osseous lesions in certain cases. CBCT provides excellent multiplanar reconstructions of the axial images allowing distinction of even the most subtle osseous changes. CBCT shows a promising potential to replace conventional radiography facilitating a precise diagnosis of maxillofacial bone lesions and operative planning.

\section{References}

[1]. Topazian R G. Osteomyelitis of jaws. In Topazian R G, Goldberg M H (eds). Oral and maxillofacial infections, Philadelphia, PA: Saunders, 1994, 3rd ed. pp 251-286.

[2]. Ronai A, Olasz L, Mulh D. Lethal complication of an odontogenic infection developing after tooth extraction in a patient with untreated diabetes-Case report. Fogorv Sz 2001;94:27-31

[3]. Marx R E. Chronic osteomyelitis of the jaws. Oral Maxillofac Surg Clin North Am 1991; 3: 367

[4]. Jacobsson S. Diffuse sclerosing osteomyelitis of the mandible. Int J Oral Surg 1984; 13: 363-385.

[5]. Schulze D., Blessmann M., Pohlenz P., Wagner K. W., Heiland M. Diagnostic criteria for the detection of mandibular osteomyelitis using cone-beam computed tomography. Dentomaxillofacial Radiology. 2006; 35(4):232-235

[6]. Taori KB, Solanke R, Mahajan SM, Rangankar V, Saini T. CT evaluation of mandibular osteomyelitis. Indian J Radiol Imaging $2005 ; 15: 447-451$

[7]. Sukovic P. Cone beam computed tomography in craniofacial imaging. Orthod Craniofac Res 2003; 6: 31-36.

[8]. Lifeng Yu, Xin Liu, Shuai Leng, James M Kofler, Juan C Ramirez-Giraldo, Mingliang Qu, et al. Radiation dose reduction in computed tomography: techniques and future perspective. Imaging in medicine. 2009; 1(1):65-84.

[9]. Boeddinghaus R, Whyte A. Current concepts in maxillofacial imaging. European Journal of Radiology, 2008; 66(3):396-418

[10]. Guttenberg SA. Oral and maxillofacial pathology in three dimensions. Dent Clin North Am 2008; 52:843-873

[11]. Pineda C, Espinosa R, Pena A. Radiographic Imaging in Osteomyelitis: The Role of Plain Radiography, Computed Tomography, Ultrasonography, Magnetic Resonance Imaging, and Scintigraphy. Seminars in Plastic Surgery. 2009;23(2):80-89. doi:10.1055/s$0029-1214160$ 
[12]. Akkas İ, Tozoglu S, Caglayan F, Özan F. The importance of CBCT imaging to determine the characteristics of a bone sequestrum in a case of chronic osteomyelitis. Cumhuriyet Dent J 2013;16(2):138-143

[13]. Hashimoto K, Arai Y, Iwai K, Araki M, Kawashima S, Terakado M. A comparison of a new limited cone beam computed tomography machine for dental use with a multidetector row helical CT machine. Oral Surg Oral Med Oral Pathol Oral Radiol Endod 2003; 95:371-377.

[14]. Ida M, Tetsumura A, Kurabayashi T, Sasaki T. Periosteal new bone formation in the jaws. A computed tomographic study. Dentomaxillofac Radiol 1997:26:169-176

\section{Legends}

\section{Figure-1}

a. Bony hard swelling with an extra oral sinus tract (white arrow)

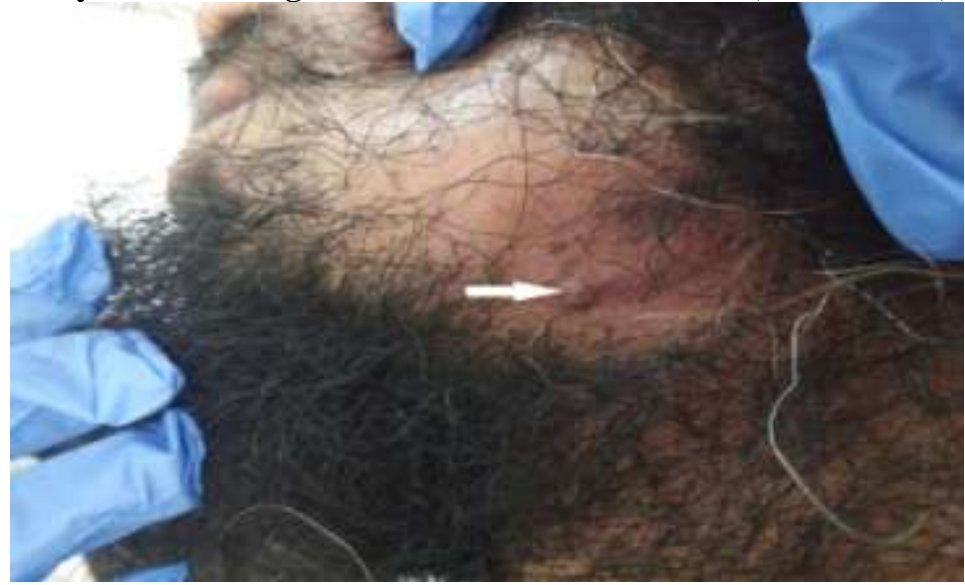

b. Submerged and carious 37 with acutely dilacerated roots and an ill-defined periapical radiolucency involving 36, 37

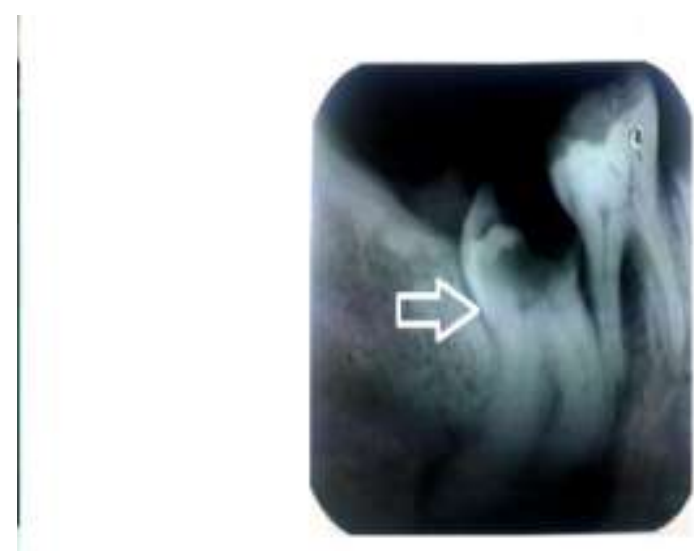

Figure-2

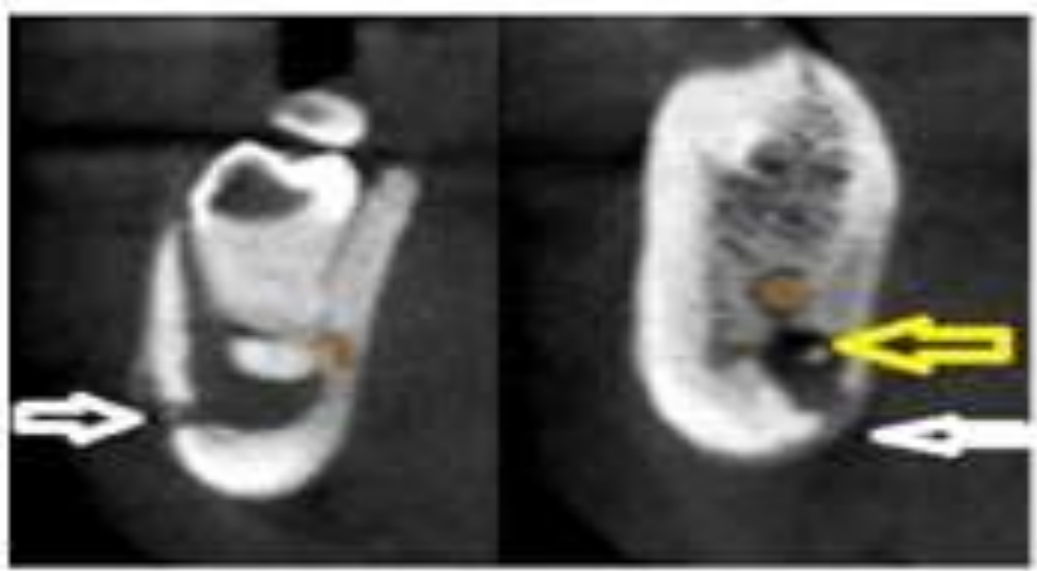


Sagittal and coronal sections showed sequestra (yellow arrow )and perforation of buccal and lingual cortex.(white arrows )

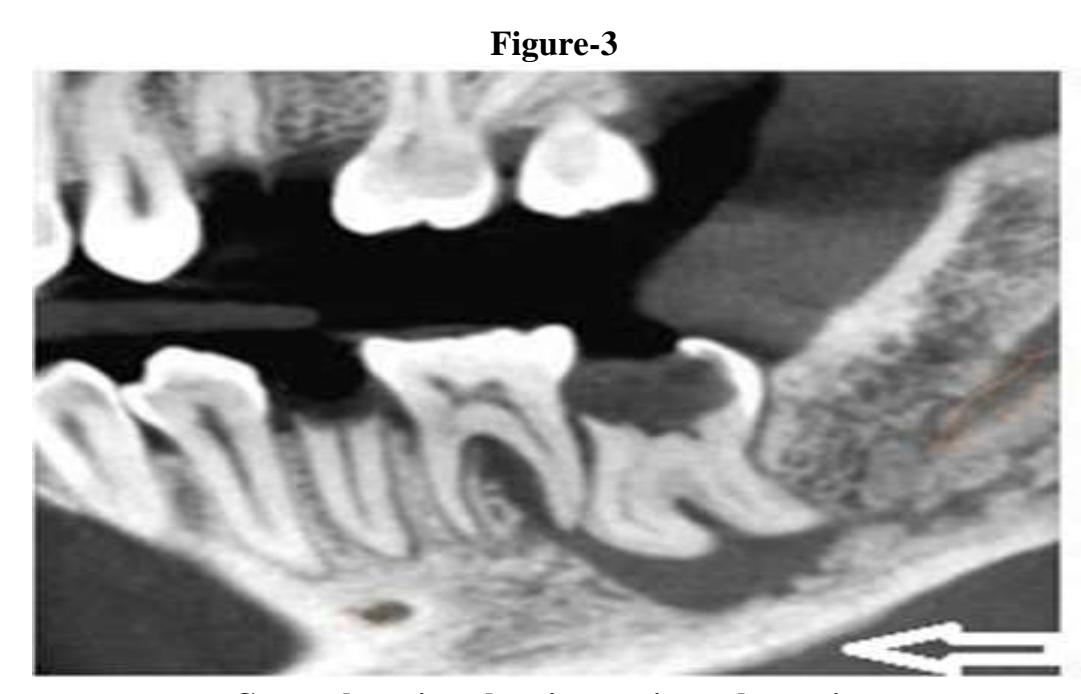

Coronal section showing periosteal reaction

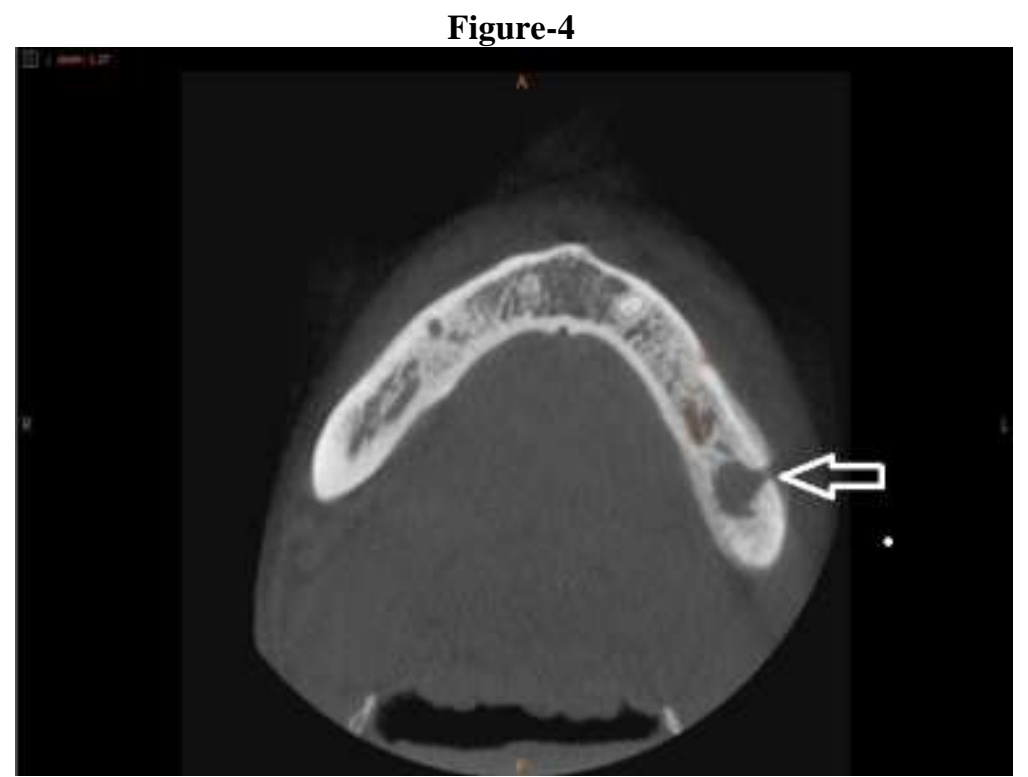

Axial section showing periosteal reaction 